\title{
Influencia de los sedimentos de las quebradas en el desarrollo de las formas de erosión
}

\section{(Influence of sediment from the Gullies in the development of erosion forms)}

\author{
Roman Krávchenko ${ }^{1}$
}

\begin{abstract}
Resumen:
En el presente artículo se exponen los procesos del desarrollo de siete quebradas. El área de estudio está ubicada en la provincia de Pichincha donde en estas quebradas se evidenciaron extensos depósitos sedimentarios. La característica de los depósitos, su estratificación y el análisis de la materia orgánica a diferentes profundidades demuestran que los depósitos se formaron a través de la acumulación en las quebradas. A una profundidad de $225-230 \mathrm{~cm}$ los depósitos tienen mayor contenido de materia orgánica que las capas situadas a más de un metro encima. Se ha investigado la influencia de los depósitos, la transformación de las formas de las quebradas y las características del proceso erosivo-acumulativo. Además se estudiaron las características de las quebradas de niveles múltiples. Las quebradas que están en la parte alta de la ladera, pertenecen a períodos anteriores a la activación de la erosión, si se compara con las quebradas en las partes bajas de ladera. Se consideró el avance cíclico de autodesarrollo de las quebradas.
\end{abstract}

Palabras clave: Quebrada; depósito sedimentario; erosión regresiva; acumulación.

\begin{abstract}
:
This article presents the process of development of seven gullies. The area of study is located in the province of Pichincha. These gullies show extensive sedimentary deposits. The characteristic of the deposits, th stratification and analysis of the organic matter at different depths, demonstrate that the deposits were formed by the accumulation in the gullies. At a depth of $225-230 \mathrm{~cm}$ the deposits have higher organic matter content than the layers located more than a meter above. The influences of the deposits, the transformation of the forms of the gullies and the characteristics of erosive-accumulative process have been investigated. Also the characteristics of the gullies at multiple levels have been studied. The gullies that are at the top of the hillside belong to periods prior to the activation of the erosion, if compared to gullies in the lower parts of mountainside. Cyclical progress was considered in the selfdevelopment of the gullies.
\end{abstract}

Keywords: Gullies; sedimentary deposits; regressive erosion; accumulation.

\section{Introducción}

La erosión por el agua es uno de los problemas globales que afectan al suelo del Ecuador. Como el resultado de la erosión del suelo se reduce su fertilidad. Las formas lineales de la erosión están destruyendo tierras de cultivo y representan amenazas para carreteras y diferentes obras de infraestructura.

\footnotetext{
${ }^{1}$ Universidad Tecnológica Equinoccial, Facultad de Ciencias de la Ingeniería, Quito - Ecuador (kra98195@ute.edu.ec)
} 
El Ecuador se caracteriza por intensivos procesos de erosión hídrica. En gran parte de su territorio existen rasgos que facilitan la erosión: cambios en la elevación, presencia de rocas sedimentarias que se parten fácilmente y clima propicio para la formación de flujos temporales de agua torrencial debido a las intensas lluvias. La actividad económica local también puede intensificar los efectos negativos de la erosión por el agua.

Según (Маккавеев, 1984; Скоморохов, 1990 et al) la erosión y la acumulación son parte del mismo proceso y se encuentran en interacción mutua. El presente estudio tiene como objetivo conocer la Influencia de los sedimentos de las quebradas en el desarrollo de las formas de erosión.

Sin embargo, el estudio de las formas de relieve, tales como quebradas, pone énfasis en el crecimiento de sus partes altas, ya que justamente la erosión regresiva produce la degradación de la tierra fértil y otros impactos negativos. No obstante, el aspecto de la influencia del proceso de la acumulación en el desarrollo de las quebradas aún permanece sin ser estudiado suficientemente.

Los estudios de la erosión y características geomorfológicas del territorio ecuatoriano han sido objeto de varias investigaciones y estudios (Argüello et al, 2012; Córdoba \& Novoa, 1995; Riesgos Naturales..., 1989; De Noni G. \& Trujillo G., 1986; Winckell, A., 1982 et al). En estos trabajos se abordaron distintos aspectos del desarrollo de las quebradas, erosión por el agua y procesos fluviales. Sin embargo, en el Ecuador es poco estudiado el tema de los depósitos en las quebradas y su impacto sobre la dinámica de las formas lineales de la erosión por el agua.

En estudios anteriores (R. Krávchenko, 2003), se examinó el rol del proceso de acumulación en el desarrollo de sistemas de quebradas en el caso de las zonas sujetas a la erosión por el agua en el norte de Eurasia. En el presente trabajo se continúa la investigación de este tema con el propósito de estudiar los depósitos de las quebradas y su impacto en el desarrollo de formas de erosiones lineales en Ecuador.

La quebrada refuerza la capacidad destructiva de las corrientes del agua. Sin embargo, el flujo del agua, saliendo a la parte baja de la quebrada y después de la salida, se aplana y deposita el material transportado. La capacidad de destrucción y transportación del flujo disminuye drásticamente en las zonas con fondo ancho y plano, donde se produce la sedimentación activa de materiales transportados.

La forma de la quebrada cambia con el tiempo. La erosión regresiva ocasiona el desplazamiento de la parte superior de la quebrada a lo largo de la ladera y la formación de nuevas partes con fondo estrecho y de gran profundidad. Pero, al mismo tiempo, sobreviene la acumulación regresiva: los sedimentos se depositan en la parte baja de la quebrada llenándola y, esta zona de acumulación activa, va avanzando gradualmente hasta la parte alta de la quebrada. 
Cuando una quebrada finaliza su crecimiento por efecto de la erosión regresiva, los procesos de acumulación continúan y pueden llevar al llenado parcial o incluso total de la quebrada. Entonces la quebrada puede dejar de existir quedando como forma de depresión de relieve. Así, los procesos erosivos-acumulativos cambian la forma de la quebrada, pero una forma de relieve transformada que produce cambios en las características y la intensidad de los procesos fluviales.

Muy a menudo después de su llenado parcial la quebrada pierde la actividad erosiva y desarrolla vegetación en su superficie y cuando el crecimiento de una quebrada se detiene, sus taludes se aplanan, llenando la parte baja de la quebrada, a veces la parte media y parcialmente la parte alta de la quebrada adquiere una forma suave.

Al término de la primera fase de acumulación, que se produce rápidamente, llega una etapa de crecimiento lento, del llenado de la quebrada, lo que puede durar cientos de años, dependiendo de su tamaño.

Al parecer, algunas de las quebradas más grandes no pueden llenarse completamente sin un cambio significativo del ambiente de sedimentación. Sin embargo en una quebrada que deja de crecer siempre hay posibilidades potenciales de reanudación de la erosión.

\section{Materiales y métodos}

El estudio se llevó a cabo en el norte de Quito, provincia de Pichincha, cantón Calderón, cerca del barrio Zabala. En el área del estudio en la Figura. 1, la flecha indica la zona de estudio detallando los depósitos de las quebradas en las siguientes coordenadas: UTM 17M789057 9991583 a una altura de 2671 m.s.n.m. Para determinar la ubicación de los puntos de investigación, sus coordenadas y alturas se utilizó el G.P.S. Se recurrió a diferentes fuentes cartográficas, incluyendo imágenes satelitales. El estudio se realiza en siete quebradas de la zona.

En las investigaciones de campo, se estudió el micro-relieve y las características morfométricas de las quebradas. El estudio se enfocó también en los depósitos de las quebradas y en sus laderas. Fueron marcados los pozos para el estudio de perfiles geológicos y de suelos y también se realizó la excavación con barreno de perforación. Los análisis del suelo y de los depósitos se realizaron en el Laboratorio de Química Agrícola y Suelos “Julio Peñaherrera" de la Universidad Central del Ecuador, Facultad de Ciencias Agrícolas; Quito - Ecuador. 


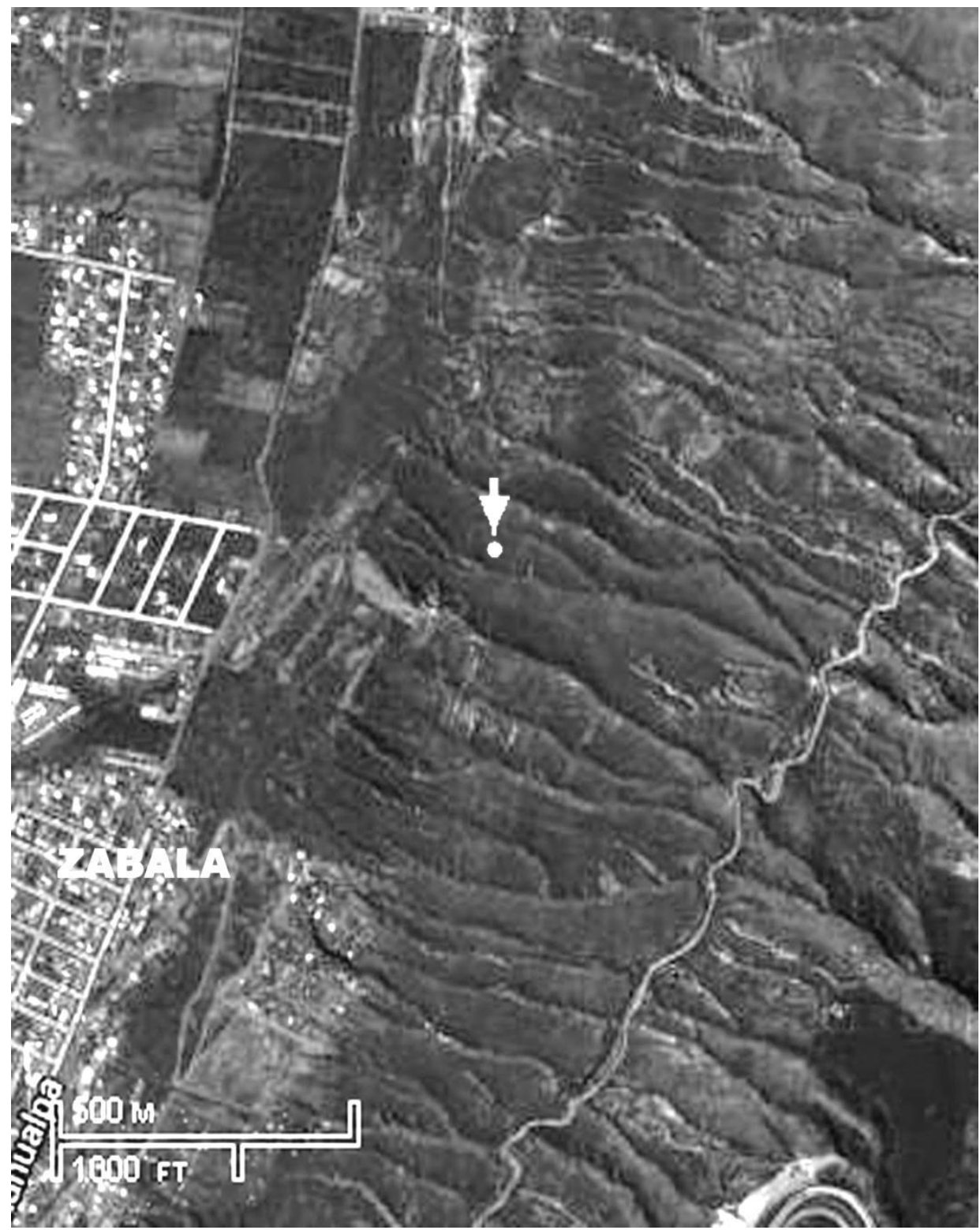

Figura 1. Área del estudio de las quebradas.

\section{Resultados y Discusión}

El área del estudio se caracteriza por un amplio desarrollo de diferentes quebradas con longitud, ancho, profundidad y grado de erosión, variables. Las quebradas que han sido estudiadas con mayor detalle se indican en la Figura 2 y representan mayor importancia para lograr el objetivo de la investigación. La Figura 2 es parte del territorio de la Figura 1.

La quebrada 1 tiene una longitud de 23,5 metros, el ángulo de inclinación de la ladera en el sitio del desarrollo de la quebrada es de 11 grados. En su parte superior la quebrada tiene la profundidad de 1,85 m, con el ancho de 1,7 m, tiene un perfil transversal en forma de cañón con fondo estrecho, la quebrada está activa y crece mediante el desplazamiento de su cabecera a lo largo de la ladera. En la parte baja la quebrada tiene los siguientes parámetros: el ancho es de $5,3 \mathrm{~m}$ y su profundidad alcanza 0,4 $\mathrm{m}$. En este lugar se realizó el estudio de los sedimentos de la superficie del fondo de la quebrada hasta la profundidad de 2,4 m. 


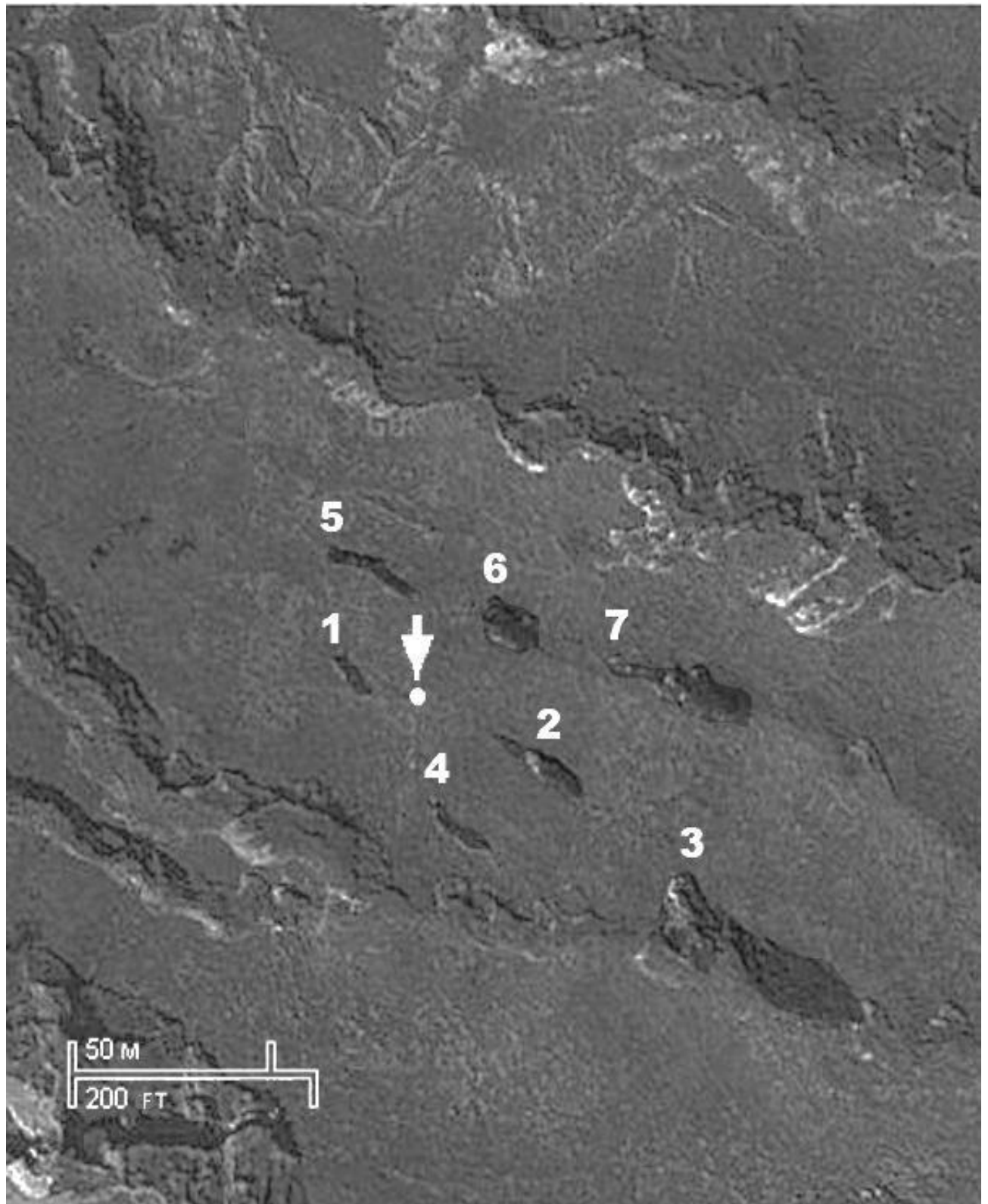

Figura 2. Representación detallada de la parte del territorio descrito en la figura 1. La flecha indica la zona (correspondiente a la Figura 1) de estudios detallados de los depósitos de las quebradas. Los números indican las quebradas, cuyas características se exponen en el artículo, y se colocan en la cúspide de cada quebrada.

La Figura 3 muestra el perfil de los depósitos. Se puso de manifiesto, que bajo la superficie del fondo de la quebrada hay sedimentos modernos que se acumularon como el resultado de flujo del agua.

Esto confirma claramente que los depósitos son estratificados, lo que nos dio claro ejemplo del desarrollo de la quebrada desde la roca madre sedimentaria dominada por partículas de arcilla, esta roca madre es de color amarillo claro. 


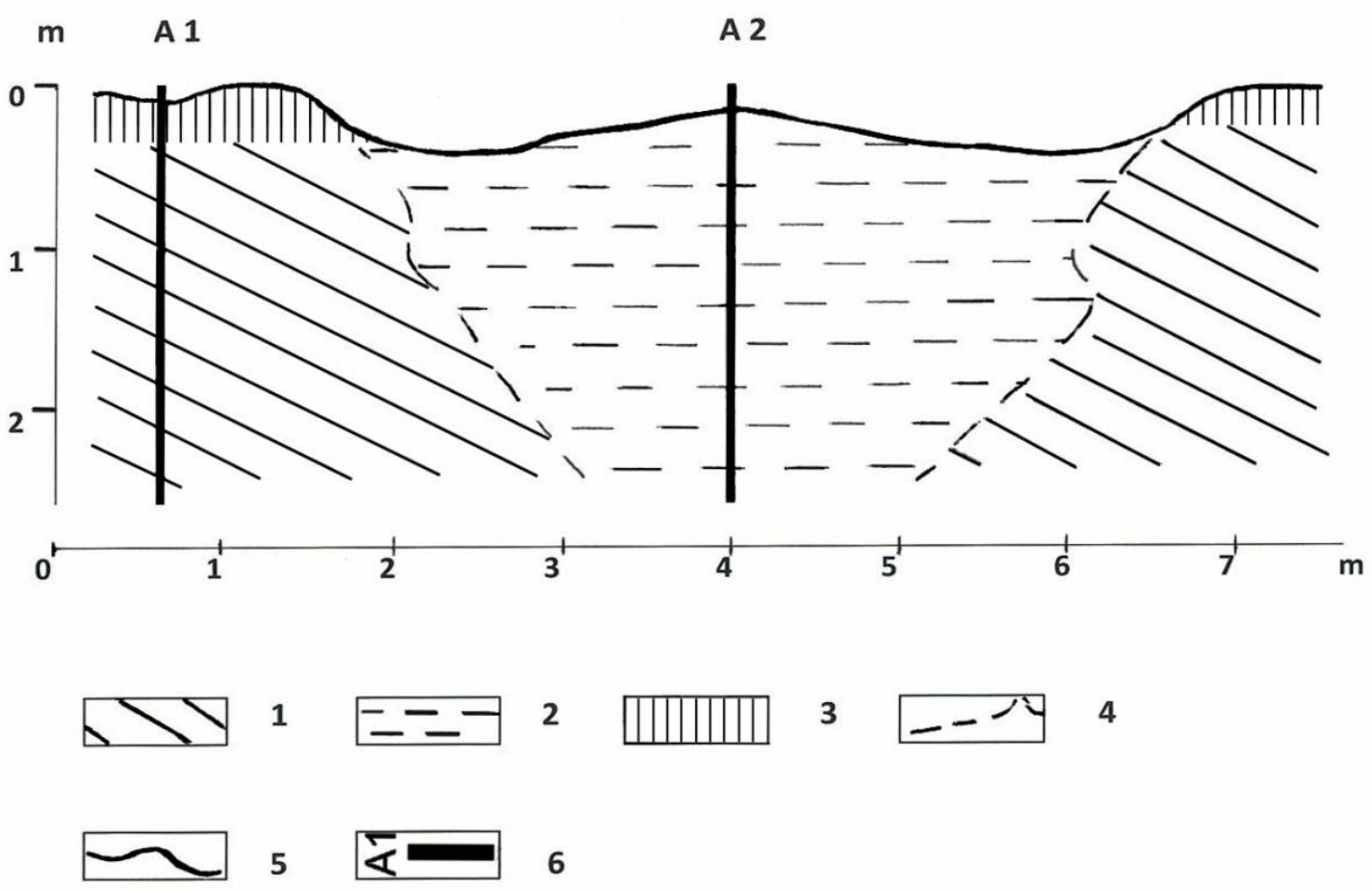

Figura 3. La sección transversal de la parte baja de la quebrada 1 y de sus depósitos. Su ubicación se presenta en las figuras 1 y 2 por punto blanco al que apunta la flecha. Símbolos de la Figura 3: 1 - Roca madre sedimentaria de color amarillo claro, dominada por partículas de arcilla. 2 - Depósitos sedimentarios estratificados de la quebrada. 3 - Suelo de las laderas. 4 - Límite entre la roca madre y los depósitos estratificados de la quebrada. 5 - Superficie de la tierra. 6 - Lugares de toma de muestras de los depósitos y suelos para su análisis.

Se establecieron diferencias entre los depósitos del fondo de la quebrada y de las zonas circundantes de la ladera, la estratificación está más clara desde la profundidad de $84 \mathrm{~cm}$, donde se observa la alternación de capas de arena de color gris oscuro y de los depósitos arcillosos de color gris, con presencia de materia orgánica.

Las capas de arena se encontraron entre las siguientes profundidades: de 84 a $97 \mathrm{~cm}$, de 111 a $118 \mathrm{~cm}$, de 121 a $135 \mathrm{~cm}$ y de 138 a $190 \mathrm{~cm}$., la estratificación refleja flujos de diferente intensidad y de diferente forma de la quebrada. El límite entre los depósitos de la quebrada y la roca madre en la misma profundidad de la ladera no está bien delimitado.

Probablemente, al mismo tiempo con el proceso de la acumulación fluvial se desarrollaron otros procesos. Así los depósitos coluviales (productos del derrumbamiento de las laderas) formaron una transición suave entre los depósitos de la quebrada y la roca madre. Además, se encontraron depósitos eólicos cuya formación es particularmente activa en el período de Junio a Septiembre.

Posteriormente gran parte de estos depósitos eólicos han sido incluidos en los procesos de transportación y sedimentación por los caudales y su sedimentación en cauce de la quebrada. 
Los resultados del análisis de la materia orgánica son una prueba más de que la forma del relieve visible en la parte baja de la quebrada con fondo ancho y poca profundidad donde se ha formado como el resultado del llenado del cauce de la quebrada (ver Tabla 1).

Tabla 1. Contenido de materia orgánica en los depósitos de la parte baja de la quebrada № 1.

\begin{tabular}{|c|c|c|}
\hline $\begin{array}{l}\text { Profundidad (desde } \\
\text { la superficie), cm }\end{array}$ & $\begin{array}{l}\text { Contenido de mat } \\
\text { toma de muestras }\end{array}$ & $\begin{array}{l}\text { S (A } 1 \text { y A } 2) \text { de } \\
\text { tra en la Figura } 3 .\end{array}$ \\
\hline & A1 & A2 \\
\hline $15-20$ & 1.06 & 1.70 \\
\hline $40-45$ & \multirow{7}{*}{0.12} & 0.76 \\
\hline $70-75$ & & 1.00 \\
\hline $100-105$ & & 0.52 \\
\hline $118-121$ & & 0.13 \\
\hline $150-155$ & & 0.07 \\
\hline $195-200$ & & 0.12 \\
\hline $225-230$ & & 0.50 \\
\hline
\end{tabular}

En el lugar de la toma de muestras A1 el contenido de la materia orgánica en roca madre no varía con la profundidad $(0,12 \%)$, mientras que la línea $A 2$ se caracteriza por alternación de las capas con diferentes contenidos de materia orgánica. Las capas de arena con el indicador mínimo de 0,07\% se intercalan con los depósitos que tienen otros indicadores.

Particular atención debe prestarse al hecho de que en la parte inferior del perfil, a una profundidad de 225 a $230 \mathrm{~cm}$, los depósitos son de color marrón oscuro y tienen mayor contenido de materia orgánica que las capas situadas a más de un metro por encima de esta capa, lo que demuestra que esta capa se formó por partículas del suelo que posteriormente fueron cubiertas por otros depósitos de acumulación del agua, incluyendo capas de arena.

Así, anteriormente está quebrada era más profunda y tenía diferente forma en su perfil transversal, pero posteriormente se llenó de depósitos. Como es bien conocido, el perfil transversal en forma de "V" contribuye a la concentración de flujo y el aumento de su capacidad de destrucción y transportación, dicha forma del perfil es típico para la parte alta de la quebrada.

En la Figura 2 llama la atención la ubicación de algunas quebradas, que están alineadas desde la parte superior hacia abajo a lo largo de la ladera. Al sur de la quebrada 1 se ubica la quebrada 2, un poco más al sur de la ladera alineada se acerca la cúspide de la quebrada 3 . Se podría 
decir entonces que, la ubicación de las quebradas es en niveles múltiples. Las quebradas 5, 6 y 7 se ubican en forma similar. En la zona entre la parte sur de la quebrada 1 y de la cabecera de la quebrada 2 según los resultados de perforación, se encuentran fragmentos de depósitos de las quebradas, lo que indica que anteriormente existía el cauce de la quebrada y su sedimentación a dado estos resultados. La Figura 4 representa el esquema del desarrollo de las quebradas de niveles múltiples, a menudo en la naturaleza se observa que la parte alta de la quebrada del nivel más bajo se desvía del cauce de la quebrada más antigua que se ubica más arriba por la ladera.

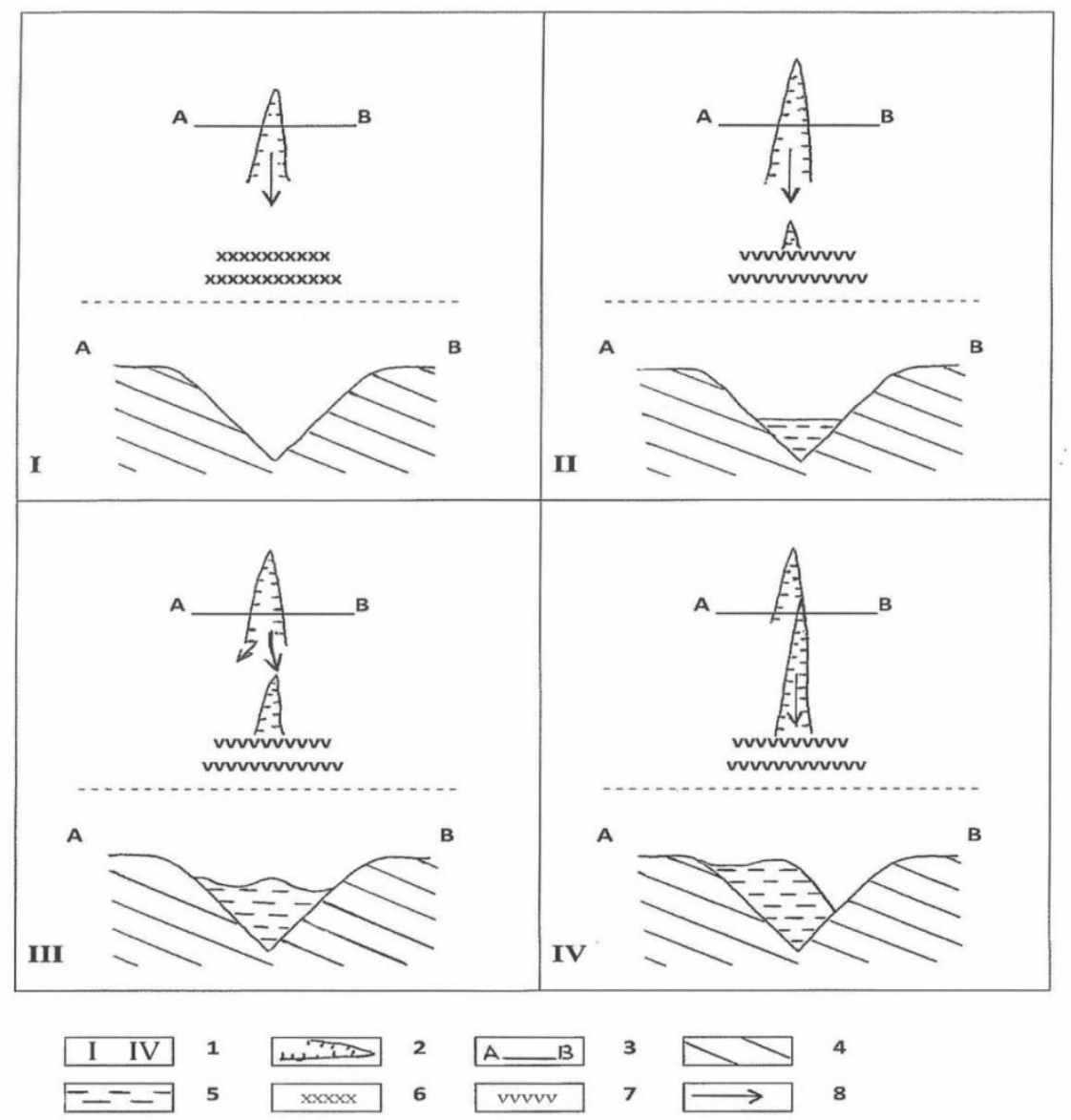

Figura 4. El esquema del desarrollo de las quebradas de niveles múltiples.

Símbolos de la Figura 4: 1 - Distintas etapas del desarrollo. 2 Quebrada. 3 - Línea del perfil transversal de la quebrada y sus depósitos la que se mantiene sin cambios en distintas etapas del desarrollo. 4 - Roca madre. 5 - Depósitos estratificados de la quebrada. 6 - Nivel de base local de la erosión. 7 - Nivel de base local de la erosión en proceso de reducción. 8 - Dirección del flujo de agua.

La razón de esta desviación es las siguiente: sobre la base de estudios de campo y de laboratorio se ha establecido (Hooke \& Rohrer, 1979) que el intenso flujo de agua se desvía hacia la periferie del cono de deyección. 
En la figura 2 se demuestra claramente cómo la parte alta de la quebrada 4 se desvía hacia el lado de un talud de la quebrada 1. El flujo de la quebrada va saliendo a su parte baja, como si se deslizara hacia el cono de deyección, porque se ubica a mayor altura.

\section{Conclusiones}

Considerando el proceso del desarrollo de las quebradas en múltiples niveles podemos llegar a las siguientes conclusiones:

Las quebradas comienzan su desarrollo a partir de la parte baja de la ladera como el resultado de cambio (reducción) del nivel de base local de erosión. Posteriormente la quebrada se desplaza hacia arriba por la ladera aumentando el alto de su cúspide. Pero al mismo tiempo, debido a la acumulación regresiva, el fondo de la quebrada va llenándose. Si, el alargamiento de una quebrada a raíz de la erosión regresiva, es igual a su acortamiento por acumulación regresiva, la quebrada empieza a moverse hacia arriba por la ladera sin cambiar su longitud.

Cabe señalar que la dinámica de las quebradas descrita puede durar décadas o siglos dependiendo de las condiciones naturales y antropogénicas. El cambio subsiguiente del nivel de base local, lleva a la formación de una nueva quebrada en la parte inferior de la ladera. Se podría decir que la quebrada recién formada "sigue" a la más antigua.

Cuando en las quebradas de niveles múltiples, la velocidad de llenado de la quebrada del nivel alto (la quebrada ubicada por encima de la ladera) es mayor que la velocidad de crecimiento de la quebrada del nivel bajo, la quebrada de formación más reciente se desarrolla en el lugar de la quebrada que se formó anteriormente y al momento se encuentra completamente llena.

Si la forma de la erosión lineal del nivel bajo destruye con su cúspide la parte baja del nivel alto, procede su fusión y activación De este modo, en el desarrollo de las quebradas podemos observar un cambio permanente de predominancia de la erosión o la acumulación.

Mientras el ciclo íntegro del desarrollo de las quebradas aún no se completa (la etapa final sería un llenado total de las formas de erosión), ya empiezan a surgir procesos de una nueva erosión. Una onda erosiva sigue a la onda erosiva anterior, por tanto las quebradas se desarrollan mediante su retorno al estado original. Sin embargo la similitud completa no es posible debido a los cambios que surgen inevitablemente en comparación con ciclos anteriores del desarrollo.

La erosión va siempre acompañada de la acumulación, en el desarrollo de las quebradas juega un papel importante no solo en la erosión regresiva, sino también la acumulación regresiva. Los depósitos sedimentarios de las quebradas, llenan las formas lineales de la erosión y afectan el flujo de agua.

La acumulación regresiva transforma el relieve y reduce la actividad erosiva, pero al mismo tiempo la acumulación crea condiciones previas para el desarrollo de una nueva erosión. 
Como resultado de ambivalencia interna del proceso erosivo-acumulativo, avanza el autodesarrollo cíclico y la auto-regulación de las quebradas, este proceso, en sus características cualitativas, es similar en las diferentes condiciones naturales en el norte de Eurasia y Ecuador.

\section{Bibliografía}

Argüello, A., Arboleda, D., Menoscal, J., Maldonado, D., \& Urresta, S., (2012, diciembre). Monitoreo de la reforestación en las quebradas en el Norte de Quito. Enfoque UTE, V.3-N.2: pp.42-63.

Córdoba, J., \& Novoa H., (1995). Problemática, experiencias y enfoque sobre la erosión, manejo y conservación de suelos de ladera en Ecuador. Seminario, IICA, Lima (Perú). Programa Cooperativo de Investigación y Transferencia de Tecnología Agropecuaria para la Subregión Andina-PROCIANDINO. Lima.

De Noni, G., \& Trujillo, G. (1986). La erosion actual y potencial en Ecuador: localizacion, manifestaciones y causas. Documentos de Investigacion, CEDIG, N 6: p. 5-14.

Hooke, R.B., Rohrer, W.L. (1979). Geometry of alluvial fans: effect of discharge and sediment size. Earth Surface Process and Landforms. N 2: - p.147-166.

Riesgos Naturales en Quito: Lahares, Aluviones y Derrumbes del Pichincha y del Cotopaxi. (1989). Quito: Corporaciòn Editora Nacional

Winckell, A. (1982). Relieve y Geomorfología del Ecuador. Documentos de Investigacion, CEDIG, N 1: p. 3-19.

Кравченко Р.А. (2003). Стадия аккумуляции в развитии овражных систем и защита земель от эрозии. Курск: Изд-во Курск. гос. ун-та, 2003.

Маккавеев (1984). Н.И. Взаимная связь процессов эрозии и аккумуляции. Эрозионные процессы (геогр. Наука - практике, под ред. Н.И.Маккавеева, Р.С.Чалова). (С. 9-11). Москва: Мысль.

Скоморохов А.И. (1990). О возвратно-поступательном развитии фрлювиального рельефа. Геоморфология. - N 2: С.12-19. 